\title{
La renovación de los diarios digitales: rediseños y web 2.0
}

\author{
Por Javier Guallar
}

Resumen: Se describen y analizan los cambios en los webs de diarios digitales españoles entre mayo y diciembre de 2006. Los principales son: la sustitución de la barra de navegación vertical por una barra horizontal superior, el diseño para una resolución de 1024 píxeles y el avance desigual en servicios de participación del lector. Se incluye una cronología de dichos cambios.

Palabras clave: Prensa digital, periódicos digitales, diseño, participación, web 2.0.

Title: Digital newspapers in Spain: redesign and web 2.0

Abstract: Design changes in Spanish digital newspapers between May and December 2006 are described and analyzed. Principal

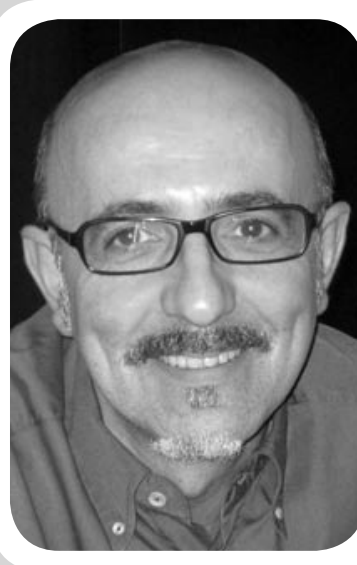

Javier Guallares licenciado en geografía e historia por la Universitat de Barcelona y licenciado en documentación por la Universitat oberta de Catalunya. Ha desarrollado su actividad profesional en varios centros de documentación de prensa. En la actualidad es documentalista del diario El periódico de Catalunya y profesor asociado de la Facultat de biblioteconomia i documentació de la Universitat de Barcelona.

changes include: substitution of the vertical navigation bar for a horizontal bar across the top of the page, design for a 1024-pixel resolution, and the uneven gains in reader services participation. A chronology of these changes is included.

Keywords: Digital press, Digital newspapers, Design, Participation, Web 2.0.

Guallar, Javier. “La renovación de los diarios digitales: rediseños y web 2.0”. En: El profesional de la información, 2007, mayo-junio, v. 16, n. 3, pp. 235-242.

DOI: 10.3145/epi.2007.may.08

2006 HA SIDO UN AÑO DE CAMBIOS VISIBLES EN LOS DIARIOS DIGITALES ESPAÑLES. Si un asiduo lector de prensa online se hubiera tomado unas hipotéticas vacaciones de internet desde mayo a finales de año, a su vuelta a la Red se habría encontrado con bastantes novedades significativas.

Efectivamente, en un período de tiempo de pocos meses varios medios han presentado renovaciones de sus webs en lo que constituye una de las corrientes de cambios más importantes de la prensa digital en su corta historia.

En este artículo se analizan las principales novedades observadas. El estudio se ha hecho a partir de la visualización directa de los sitios web durante los días siguientes a la presentación de los nuevos diseños así como de las explicaciones ofrecidas por las propias cabeceras y de la consulta de fuentes especializadas en la actualidad de los medios de comunicación digitales ${ }^{1}$. Se co- menta un total de 23 rediseños detectados, entre los que se encuentra la mayoría de diarios españoles de mayor difusión en internet junto a algunos de ámbito regional (ver figura 1).

\section{Secuencia de los rediseños}

En mayo de 2006 Abc.es y Marca.com llaman la atención de muchos internautas al modificar sus sitios coincidiendo con un evento informativo de gran magnitud como es el Campeonato mundial de fútbol. Inauguran de esta manera una serie de novedades que en los siguientes meses cambiará el aspecto de buena parte de la prensa digital española.

"En pocos meses varios medios han presentado renovaciones de sus webs en lo que constituye una de las corrientes de cambios más importantes de la prensa
Aunque en realidad éstos no son los primeros cambios del año en medios de comunicación de nuestro país, ya que unos días antes dos diarios de ámbito regional y por tanto de repercusión más reducida habían presentado renovaciones. $E l$ correo gallego.es estrenaba el 1 de mayo un web diseñado para monitores de 1024 píxeles, como hacían ya desde algunos meses varios medios internacionales, en vez del utilizado mayoritariamente en España de 800 píxeles. El otro cambio es el de Diario de Mallorca.es el 16 de mayo, que aporta como principales novedades la introducción de rss y blogs, elementos ya presentes en las principales cabeceras desde hacía algún tiempo. digital” 
Abc.es y Marca.com estrenan nuevos sitios el 22 y el 31 de mayo respectivamente. Ambos coinciden en eliminar la barra vertical de navegación situada a la izquierda de la pantalla que hasta ese momento era omnipresente en los cibermedios españoles, y en sustituirla por un único menú horizontal superior. Esta innovación da lugar a una nueva distribución de la información en tres columnas, que después se verá en otros muchos casos. Así, en Abc.es la primera columna se dedica a las noticias principales, la central a contenidos de ocio, deportes y otros reportajes, y la tercera a servicios diversos como encuestas, foros o información meteorológica. Por su parte Marca.com añade una segunda novedad sustancial, la ampliación de formato con un diseño para 1024 píxeles de ancho. Aunque como se ha visto, no es el primer medio español en hacerlo, sí es el primero de los grandes, e inicia con ello el debate entre partidarios y detractores de los diseños a 800 o a 1024 píxeles.

En los días siguientes, tres cabeceras de peso coinciden en la supresión del menú lateral y su sustitución por una única barra horizontal de navegación y ya se empieza a hablar de una tendencia manifiesta en la prensa española en internet: $E l$ mundo.es (7 de junio), La vanguardia.es (13 de junio) y 20 minutos.es (20 de junio).

El nuevo aspecto de El mundo. es, que también amplía su formato, en tanto que líder en difusión tendrá una importante repercusión y su influencia es fácilmente perceptible en algunos rediseños posteriores. Este cambio no viene acompañado en esta ocasión de grandes novedades en contenidos, si bien en los meses previos ya se había distinguido con algunas innovaciones: la introducción de videos de manera destacada en las informaciones, la utilización de Google Maps en su Callejero, la incorporación de noticias relacionadas o la ampliación de la sección de weblogs.

Tanto La vanguardia.es como 20 minutos.es mantienen el formato "antiguo" de 800 píxeles pero en cuanto a contenidos son los primeros medios online en emplear un elemento característico de las tendencias web 2.0 como son las etiquetas (tags). La vanguardia.es,

\begin{tabular}{|c|c|c|c|c|}
\hline Diario & URL & $\begin{array}{c}\text { Fecha } \\
\text { presentación } \\
\text { nuevo web }\end{array}$ & $\begin{array}{c}\text { Supresión } \\
\text { barra } \\
\text { vertical }\end{array}$ & $\begin{array}{l}\text { Diseño a } \\
1024 \text { px }\end{array}$ \\
\hline El correo gallego.es & http://www.elcorreogallego.es & 1 mayo & No & Sí \\
\hline Diario de Mallorca.es & http://diariodemallorca.es & 16 mayo & No & No \\
\hline Abc.es & http://www.abc.es/ & 22 mayo & Sí & No \\
\hline Marca.com & http://www.marca.com & 31 mayo & Sí & Sí \\
\hline El mundo.es & http://www.elmundo.es & 7 junio & Sí & Sí \\
\hline La vanguardia.es & http://www.lavanguardia.es & 13 junio & Sí & No \\
\hline 20 minutos.es & http://www.20minutos.es & 20 junio & Sí & No \\
\hline El periodico.com & http://www.elperiodico.com & 5 septiembre & Sí & Sí \\
\hline El confidencial.com & http://www.elconfidencial.com & 11 septiembre & Sí & Sí \\
\hline $\begin{array}{l}\text { El correodigital.com, } \\
\text { Diario vasco }\end{array}$ & $\begin{array}{l}\text { http://www.elcorreodigital.com } \\
\text { http://www.diariovasco.com }\end{array}$ & 28 septiembre & No & No \\
\hline Periodista digital & http://www.periodistadigital.com & 12 octubre & Sí & Sí \\
\hline Canarias ahora.es & http://www.canariasahora.es & 2 noviembre & No & Sí \\
\hline $\begin{array}{l}\text { Diarios grupo Joly: } \\
\text { Diario de Cadiz.es, } \\
\text { Diario de Jerez.es, } \\
\text { Diario de Sevilla.es, } \\
\text { El Día de Cordoba.es, } \\
\text { Europa sur.es, } \\
\text { Granada hoy.com, } \\
\text { Huelva informacion.es, } \\
\text { Málaga hoy.es }\end{array}$ & $\begin{array}{l}\text { http://www.diariodecadiz.es/ } \\
\text { http://www.diariodejerez.es/ } \\
\text { http://www.diariodesevilla.es/ } \\
\text { http://www.eldiadecordoba.es/ } \\
\text { http://www.europasur.es/ } \\
\text { http://www.granadahoy.com/ } \\
\text { http://www.huelvainformacion.es/ } \\
\text { http://www.malagahoy.es/ }\end{array}$ & 4 noviembre & No & Sí \\
\hline El pais.com & http://www.elpais.com & 20 noviembre & Sí & Sí \\
\hline Diario de Navarra.es & http://www.diariodenavarra.es & 17 diciembre & Sí & Sí \\
\hline
\end{tabular}

Figura 1. Cronología de nuevos diseños de diarios digitales (mayo a diciembre de 2006) 
que ya las venía utilizando desde diciembre de 2005, añade ahora entre otras posibilidades de participación de los lectores las opiniones de éstos en determinados artículos (que llaman ahora blogs). Por su parte, 20 minutos.es lleva a cabo la renovación más radical de la prensa española con una portada que presenta las noticias con titulares a gran tamaño, sin texto y con más espacio para las imágenes, además de opciones de comentarios a las noticias, presencia destacada de bitácoras y la llamativa aparición bajo el menú horizontal de nubes de etiquetas (tags clouds).

Tras un período estival sin novedades, tres cibermedios importantes, El periódico (5 de septiembre), El confidencial (11 de septiembre) y Periodista digital (12 de octubre), y dos regionales del grupo Vocento, El correodigital.com y Diario vasco (28 de septiembre) presentan nuevos webs. Mientras que los rediseños de los dos diarios vascos son muy modestos ya que no se suman a los últimos cambios formales y sólo presentan como aspecto reseñable la agrupación en la barra superior de los elementos de participación, los otros tres sí incorporan las nuevas tendencias de menú horizontal único y de ampliación de formato. Además, $E l$ periódico, que separa los dominios de sus ediciones en castellano y en catalán ( $E l$ periodico.com y $E l$ periodico.cat), otorga mayor protagonismo a los espacios de participación del lector, y establece una relación muy directa con la edición impresa, de tal manera que las intervenciones de los usuarios de la edición online en foros, encuestas, fotocartas, etc, son trasladadas diariamente a la edición en papel en lugares destacados.

El confidencial limita su actualización a los aspectos formales señalados y a la supresión del dominio com de la cabecera, y Periodista digital estrena un sitio de as-
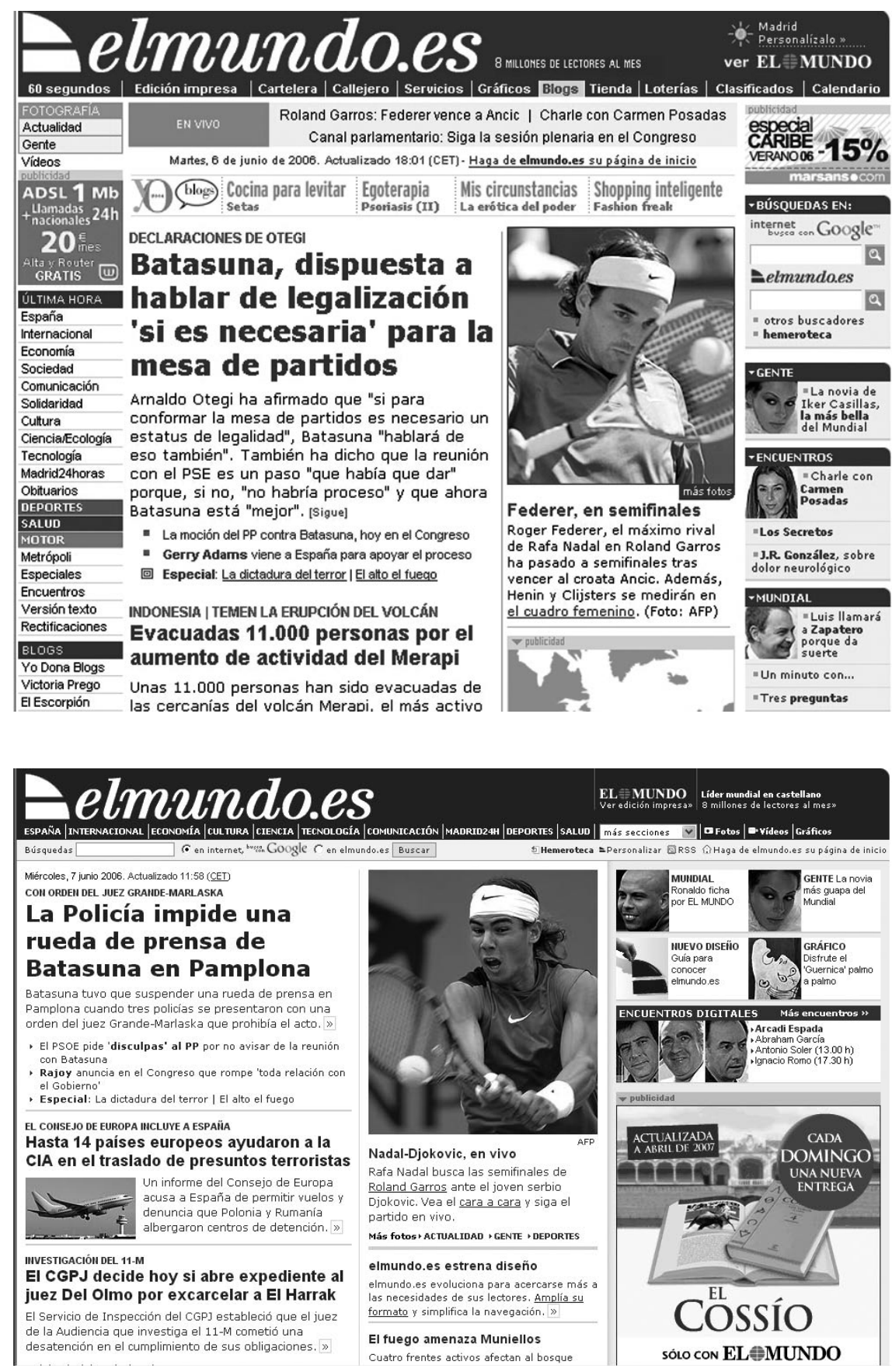

Portadas de El mundo.es antes y después del cambio (6 y 7 de junio)

pecto muy similar al de El mundo. es, con importantes avances en los servicios participativos. Continúa promoviendo la incorporación de bitácoras a su zona de blogs (blog zone), la más poblada de la prensa española, y presenta asimismo como gran novedad un proyecto de periodismo ciudadano o periodismo 3.0 denominado Reportero digital. Con este servicio, pretende expandir por España una red de ediciones locales basadas en la redacción de informaciones por parte de usuarios y coordinadas por una o dos personas de la cabecera en cada edición.

En los días siguientes, los nuevos webs de Canarias ahora (2 de noviembre) y de los medios regionales del grupo Joly: Diario de Cádiz, Diario de Jerez, Diario de Sevilla, El Día de Córdoba, Europa Sur, Granada Hoy, Huelva Información y Málaga Hoy (4 de noviembre) son, como en otros casos de cabeceras de ámbito regional, de resultados más modestos. Aunque sí amplían su formato, tanto la estructura for- 


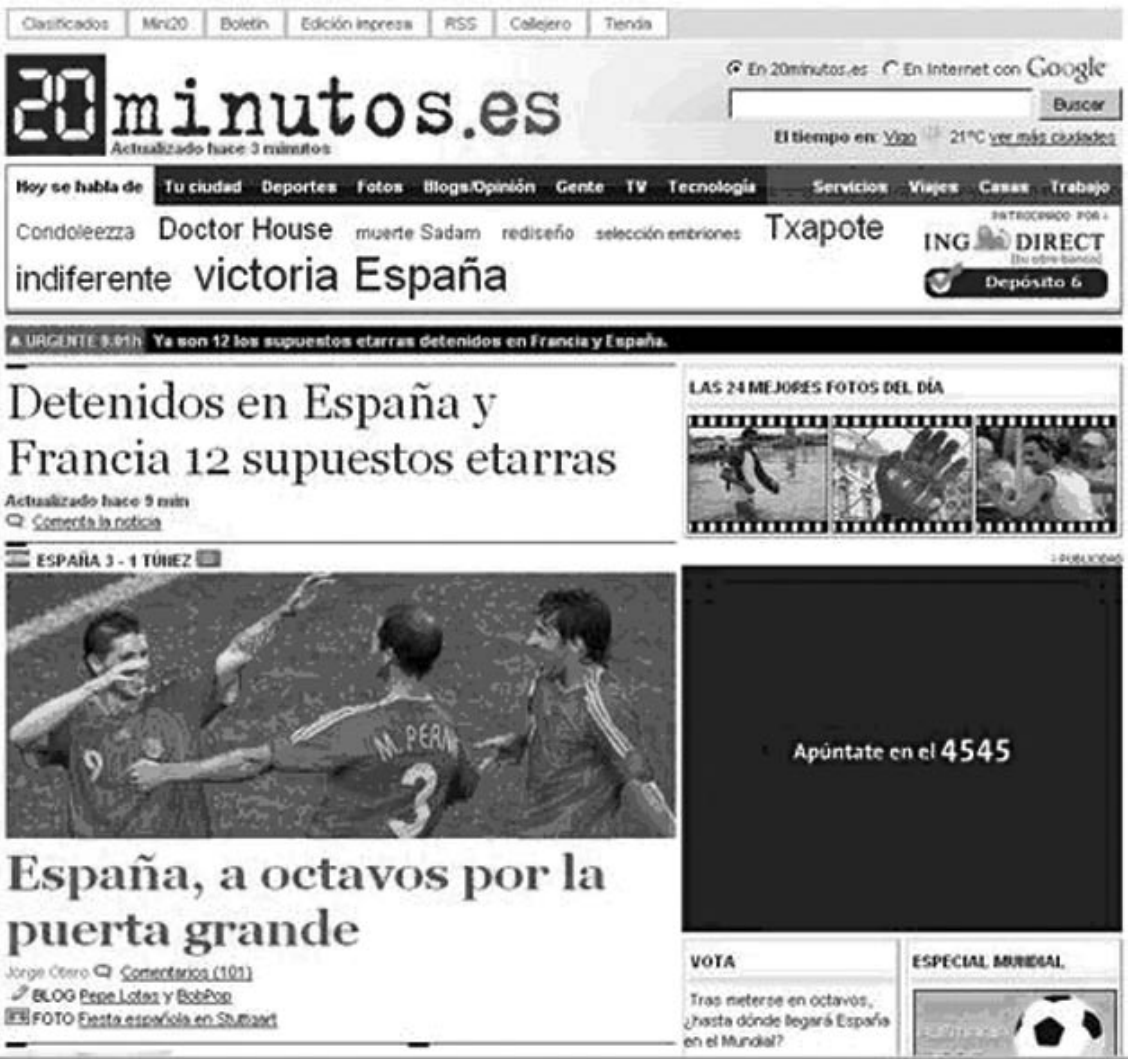

Portada de 20 minutos.es (20 de junio)

mal con la permanencia de la barra vertical como la ausencia o escasez de elementos de participación los sitúan en una etapa evolutiva anterior a la de los grandes diarios.

El ciclo de renovaciones de 2006 prácticamente se cierra el 20 de noviembre con el esperado estreno del nuevo diseño de $E l$ país, la principal competencia de El mundo. es por el liderazgo de la prensa online española. El sitio, que cambia el dominio es por el com, presenta importantes novedades. Utiliza tanto el menú horizontal único como el diseño a 1024, y tiene además la virtud de aprovechar la ampliación de formato para situar el web en el centro de la pantalla y no a la izquierda de la misma como en todos los casos anteriores. Con ello mejora la visualización al eliminar el espacio vacío de la derecha del monitor.

Los cambios en contenidos son aún de mayor calado. Hay una decidida utilización de las posibilidades multimedia con una presencia mayor de videos en la portada, así como especialmente de aplicacio- nes web 2.0. El nuevo El pais.com parece querer aprovechar cuantos recursos participativos han ensayado los rediseños anteriores. Crea una gran sección de blogs, en la línea de las ya exitosas de El mundo. es y Periodista digital y agrupa en la sección Participa algunos servicios ya existentes junto a nuevas incorporaciones, como la ordenación de noticias a partir de los votos y el apartado Yo periodista, invitación a los lectores al periodismo ciudadano. También se pueden votar las noticias, que aparecen con un número de estrellas a partir de los votos recibidos, y se incluyen enlaces en la parte inferior de cada pieza informativa a algunos de los sitios 2.0 más populares: Del.icio.us, Digg, Menéame, Mi Yahoo y Technorati. Ésto último es especialmente destacable si se tiene en cuenta que entre noviembre de 2002 y junio de 2005 El país había sido el único medio español que había decidido cerrar el acceso libre a su sitio haciéndolo completamente de pago $^{1}$. El cambio desde cerrar todos sus contenidos a promover que los lectores los en- víen a Digg o Menéame constituye ciertamente un giro de 180 grados en su estrategia en internet.

Antes de fin de año, una última renovación a reseñar: la de Diario de Navarra.es (16 de diciembre), que asume las dos tendencias formales consolidadas y añade como única novedad en servicios la aparición de fuentes rss.

\section{"La novedad más repetida en los rediseños es la supresión del menú vertical izquierdo"}

\section{Desaparición de la barra de navegación vertical}

La novedad más repetida en los rediseños estudiados es la supresión del menú vertical situado a la izquierda de la página de inicio. Ésta es una medida que ya venían empleando algunos diarios pioneros, por ejemplo Clarin.com desde la lejana fecha de noviembre de 2004, o Washington post.com desde febrero de 2005.

En el modelo anterior la barra lateral es la referencia principal de acceso a los contenidos. En ocasiones puede ser muy detallada y complementa a un menú horizontal superior de carácter más general. Con el nuevo diseño la navegación principal del sitio se traslada totalmente a la parte superior de la página y se horizontaliza en buena medida al desaparecer la necesidad de descender por el índice vertical en busca de contenidos.

La barra horizontal superior presenta a su vez variantes: una única línea (El mundo.es); subdivisión en dos líneas, una para grandes secciones y la segunda para otros contenidos (El pais.com); utilización de pestañas desplegables $(E l$ periodico.com); inclusión de tags (20 minutos.es). 


\section{"La estructura de la web sigue un modelo de 'nuevo tridente' basado en dos columnas dedicadas a noticias y una tercera de servicios, publicidad y promociones"}

Este cambio tiene consecuencias en la estructura de la información de la página de inicio. El modelo seguido mayoritariamente hasta 2006, llamado por algunos autores de tetradente (figura 2), se caracterizaba por la evolución desde el tridente original de los primeros años de la prensa online (bloque izquierdo de navegación, central de noticias y derecho de publicidad y servicios) a un tetradente (Armentia, 2005), en el que el bloque central informativo se subdivide en dos columnas generalmente de ancho diferente, para noticias principales y secundarias.

La presentación actual modifica este esquema y sigue lo que aquí denominamos modelo de nuevo tridente (figura 3) basado en dos columnas dedicadas a noticias y una tercera de servicios, publicidad y promociones. Una de las dos columnas informativas suele tener una amplitud muy superior a la otra, por ejemplo, la izquierda en El pais.com, El mundo.es, Abc.es o El periodico.com, y la central en La vanguardia.es o Periodista digital. Este esquema no impide que en ocasiones, ante un acontecimiento

informativo que se quiere remarcar especialmente, la información pueda aparecer a dos e incluso a tres columnas.

La nueva estructura genera portadas más claras, menos densas y abigarradas, se elimina la duplicidad de navegación vertical y horizontal en los casos en que existía doble menú, y el espacio que se gana con la eliminación del índice lateral se puede dedicar a presentar mejor las noticias.

\section{Diseño para resolución a 1.024 píxeles}

El segundo cambio formal importante de la temporada es la sustitución del diseño pensado para pantallas de $800 \times 600$ píxeles por uno optimizado para monitores de 1024x768 píxeles, como venían haciendo desde unos meses algunos medios norteamericanos, por ejemplo The New York times desde principios de abril. El efecto conseguido con el formato de 1024 es el de una notable ampliación del espacio que ocupa el web en el monitor.

Esta decisión guarda una relación directa con las posibilida-

\begin{tabular}{|c|c|c|c|}
\hline \multicolumn{3}{|c|}{ Cabecera } \\
\hline \multicolumn{3}{|c|}{ Menú de navegación (opcional) } \\
\hline $\begin{array}{c}\text { Menú de } \\
\text { navegación }\end{array}$ & Noticias & Noticias & Servicios y publicidad \\
\hline
\end{tabular}

Figura 2. Modelo de tetradente

\begin{tabular}{|l|l|l|}
\hline \multicolumn{3}{|c|}{ Cabecera } \\
\hline \multicolumn{3}{|c|}{ Menú de navegación } \\
\hline Noticias importantes & Noticias secundarias & Servicios y publicidad \\
\hline
\end{tabular}

Figura 3. Modelo de nuevo tridente des de visualización que permite una determinada resolución de los equipos informáticos. Sobre esta cuestión son reveladores los datos ofrecidos por OneStat. Según esta empresa consultora, en junio de 2006 el 56,2\% de monitores a nivel mundial eran de resoluciones de $1024 \times 768$ píxeles, el $15,8 \%$ de $1280 \times 1024$ y solamente el $12,0 \%$ de $800 \times 600$. Un año antes, este último porcentaje era de 18,2\% (OneStat 2006). Aunque se pueda considerar al no tener datos específicos de España que aquí la situación es algo menos acentuada, las cifras anteriores muestran con claridad la conveniencia de diseñar webs teniendo en cuenta la existencia mayoritaria de un parque de ordenadores con resoluciones altas y la tendencia decreciente de las bajas. La otra opción posible, la del denominado diseño líquido o variable, que ha sido utilizado en otros contextos (blogs y portales), es prácticamente desconocido en el ámbito de la prensa online.

\section{"El efecto conseguido con el formato de 1024 píxeles es el de una notable ampliación del espacio que ocupa el web en el monitor"}

Son mayoría los cibermedios que incorporan al mismo tiempo en sus nuevos webs las dos renovaciones formales señaladas, pero también hay excepciones en un sentido y en otro. Por ejemplo, $A b c$. es, 20 minutos.es o La vanguardia. es suprimen la barra lateral pero no aumentan el formato, mientras que los diarios regionales del grupo Joly hacen lo contrario (ver figura 1 ).

\section{Web 2.0 en la prensa digital}

Además de cambios notables en diseño y estructura, los nuevos sitios también presentan algunas 


\section{elPeriódico.com

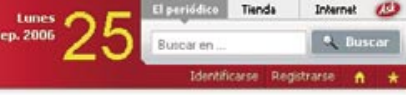

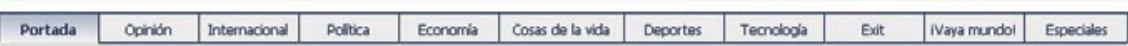
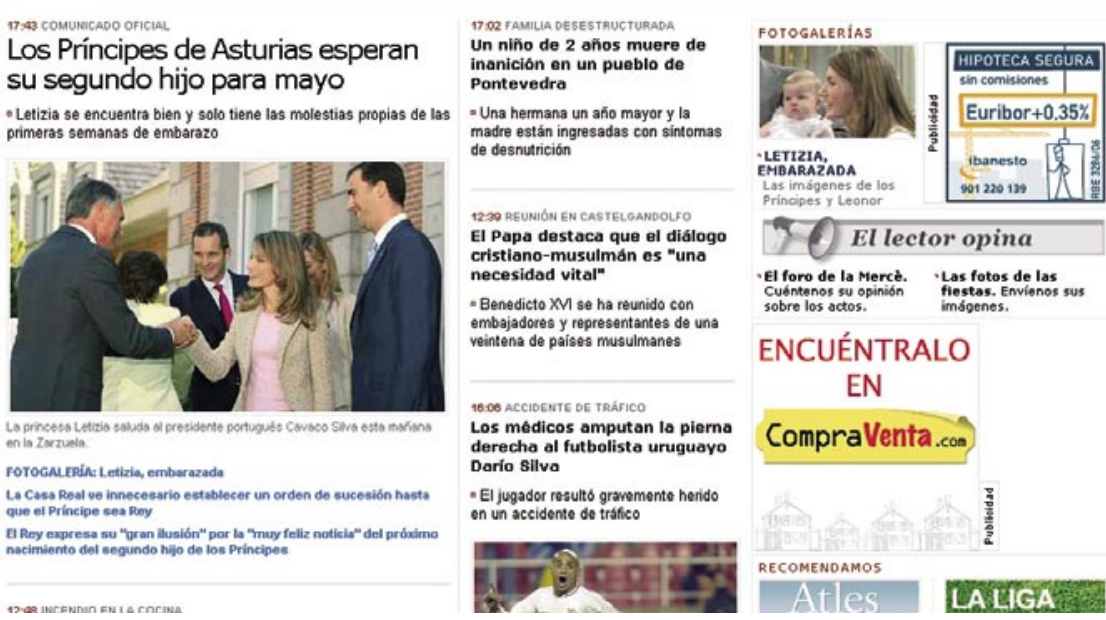

orinopes $r$ toon

70 El lector opina

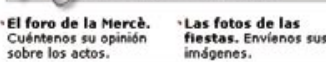

ENCUÉNTRALO

EN

CompraVenta.coo

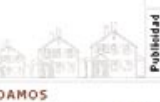
Attes LALEA

Portada de El periodico.com

modificaciones en contenidos y servicios. La tendencia visible más importante desde este punto de vista es la creciente participación del lector en el producto informativo.

Aunque la interacción entre ciudadanos y medios de comunicación en internet no es un hecho novedoso ya que hace años que éstos vienen ofreciendo los servicios denominados tradicionalmente de comunicación (foros, chats y encuestas), sí que es interesante destacar el avance participativo que se aprecia en la actualidad. Ahora se hace bien explícita la preocupación por hacer de los visitantes algo más que simples lectores, y se les invita ya no sólo a opinar sino además a participar en la presentación y la elaboración del contenido de los diarios. Los medios que realizan las actuaciones más decididas en esta línea se suman así a las tendencias que se extienden por la Red bajo la denominación web 2.0. ${ }^{2}$

\section{"Se invita a los lectores no sólo a opinar sino además a participar en la presentación y la elaboración del contenido de los diarios"}

Las posibilidades son varias aunque se materializan a niveles muy desiguales entre los cibermedios más adelantados y los que las incorporan más tímidamente. Se puede citar entre los primeros ejemplos como 20 minutos.es, El pais. com, El mundo.es, Periodista digital, Abc.es, La vanguardia.es, El periodico.com; y entre los segundos buena parte de los diarios más modestos.

Algunos de estos sistemas y servicios que permiten al lector un mayor protagonismo en su relación con el medio online son:

\section{- Secciones de blogs}

$\mathrm{Su}$ incorporación como parte del contenido informativo de los cibermedios sigue la lógica de la evolución de las tradicionales columnas de opinión de la prensa impresa adaptadas al entorno de la Red, que ahora pueden mantener un contacto más fluido con los lectores invitándolos a comentar cada artículo o post. La importancia de esta interacción posterior es clave para situar cada caso en un nivel auténticamente participativo o no. En los casos en que esto no se consigue, el supuesto blog no pasaría de ser un texto de opinión publicado en internet.

La influencia del fenómeno weblog en la prensa digital había dado lugar bien pronto a secciones específicas en aquellos diarios más conectados con las nuevas tendencias (Periodista digital, $20 \mathrm{minu}$ tos.es) hasta llegar a consolidarse con ejemplos muy exitosos como la sección de El mundo.es. En los nuevos webs de 2006, la inclusión de blogs sigue estas pautas, siendo el ejemplo más destacado el de $E l$ pais.com. Aun así, algunos diarios siguen sin incorporarlos.

\section{- Canales rss}

La utilización de sistemas de sindicación de contenidos mediante rss aparece en la prensa española en internet ya en 2003 (El mundo.es), aunque su utilización por el público ha sido escasa hasta el momento (Noguera, 2006). La mayoría de medios ofrecen esta funcionalidad, y algunos han aprovechado los nuevos diseños para incorporarla por vez primera (por ejemplo, Diario de Mallorca.es, Diario de Navarra. es).

- Valoración por los lectores de las informaciones del medio

Los diarios online empiezan a aplicar en sus sitios herramientas para la valoración y promoción de noticias por los lectores. La utilización de estos servicios hace visible la participación del usuario en la configuración final de los contenidos del medio.

Dos muestras en los rediseños de 2006 son la diversidad de elementos de valoración de las noticias en El pais.com: votación, clasificación con estrellas, envío de noticias a webs sociales como Del. icio.us, Digg, Menéame, etc.; y la nube de etiquetas (tags cloud) que 20 minutos.es sitúa de manera destacada bajo el menú principal: una relación de términos que enlazan con las noticias así clasificadas y que tienen un tamaño variable en función de varios parámetros, por ejemplo, noticias más consultadas, comentarios favorables de los lectores, enlaces, etc. 


\section{- Elaboración de informacio- nes propias por los lectores}

Son secciones dedicadas a informaciones redactadas directamente por los propios lectores, en lo que se suele denominar periodismo ciudadano o periodismo $3.0^{3}$. Aunque están poco extendidas entre los medios españoles, hay ejemplos significativos estrenados en 2006: Reportero Digital de Periodista digital, o Yo periodista de El pais.com.

\section{Conclusiones}

En 2006 muchos diarios digitales españoles han realizado renovaciones de sus webs en lo que constituye una oleada de cambios de gran magnitud, la mayor en la prensa digital en los últimos años.

Los elementos de cambio más destacados se pueden situar en dos ámbitos diferentes: el ámbito formal y el de contenidos.

En lo referente a la reestructuración formal, hay dos grandes novedades: la desaparición en la página de inicio de la barra de navegación vertical y su sustitución por un único menú horizontal superior, que a su vez da lugar a una nueva estructura de la información en tres columnas; y la ampliación del espacio que ocupa el web en la pantalla al utilizar diseños optimizados para monitores de 1024 píxeles.

En cuanto a contenidos, la tendencia más visible se orienta a la potenciación de la participación de los lectores en el producto final bajo diversas fórmulas. A los veteranos servicios de comunicación (foros, chats y encuestas), se añaden sistemas de aparición más reciente que en los casos más avanzados suelen identificarse con las tendencias denominadas genéricamente web 2.0 (blogs, rss, tags, secciones de periodismo ciudadano, enlaces a webs externos de criterio social), y representan un salto cualitativo en la concepción de las posibilidades participativas de los usuarios.

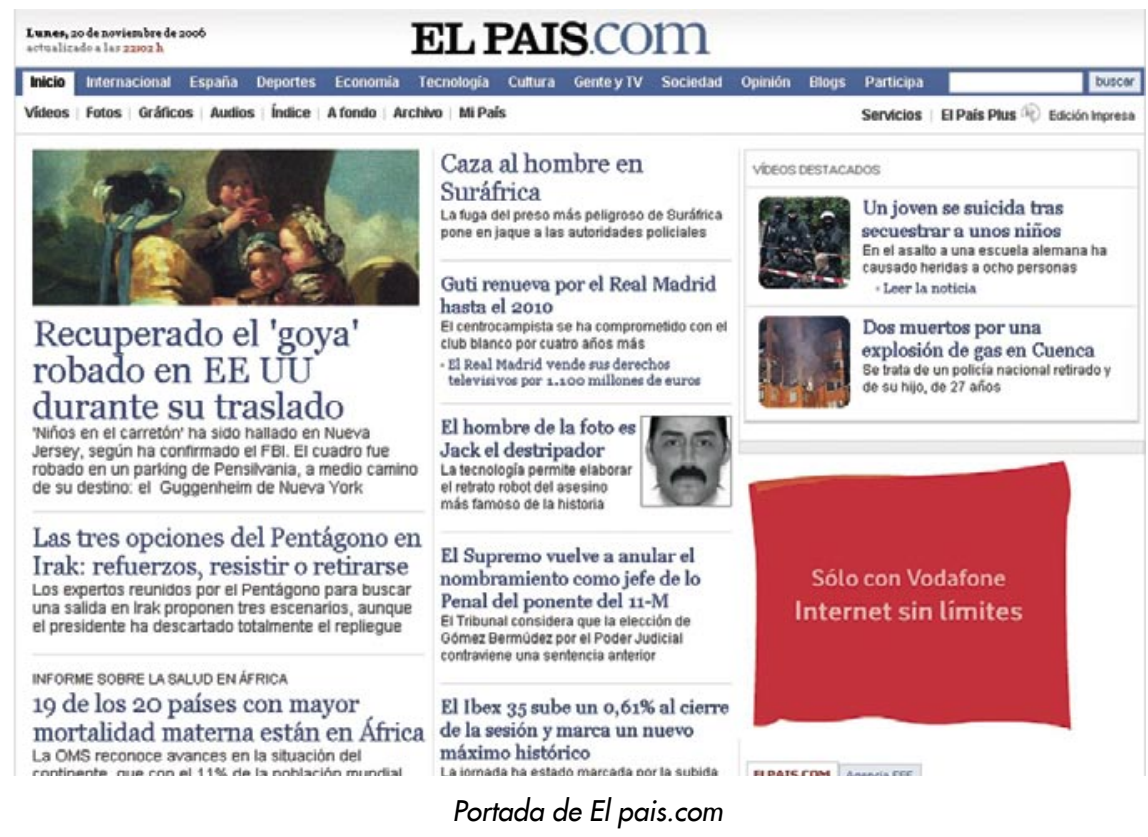

Por último, la prensa digital evoluciona pero no todos los medios avanzan al mismo ritmo. Las diferencias entre los nuevos diarios presentados en 2006 son apreciables tanto en el seguimiento de las nuevas tendencias de diseño, como especialmente en el avance de los servicios de participación del lector, donde las diferencias son más acusadas entre los sitios más activos, que han entrado de lleno en la filosofía 2.0, y los que permanecen en un estadio evolutivo anterior.

\section{Notas}

1. La mayoría de cibermedios han informado de sus rediseños a los lectores. De todas estas informaciones, se han incluido en la bibliografía aquellas que permanecían accesibles en la fecha de cierre de este artículo (27 de febrero de 2007). Asimismo se han consultado regularmente las publicaciones que comentan la actualidad de la prensa digital española, como por ejemplo los blogs e-periodistas weblog y Periodistas 21, de Ramón Salavarría y Juan Varela respectivamente. http://e-periodistas.blogspot.com/ http://periodistas21.blogspot.com/

2. Un resumen de la evolución de la estrategia de pago de El país se puede ver en el apartado "Gratuidad versus pago, el caso de El país" de Guallar (2006).

\section{"La prensa digital evoluciona pero no todos los medios avanzan al mismo ritmo"}

3. Una introducción al concepto y las aplicaciones web 2.0 se puede ver en Serrano-Cobos (2006) y Margaix (2007).

4. Un estudio con abundantes referencias sobre el concepto de periodismo 3.0 se puede consultar en Varela (2007).

\section{Bibliografía}

Alique, Rafael. "Bienvenidos de nuevo". En: Marca.com, 31 de mayo de 2006. Consultado en: 27-02-07.

http://www.Marca.com/bienvenida.html

Armentia-Vizuete, José-Ignacio. "Los diarios digitales siguen buscando su propia identidad tras una década de existencia”. En: Estudios sobre el mensaje periodístico, 2005, n.11, pp. 9-22.

Canarias ahora.es, "Canarias ahora renueva su diseño". En: Canarias ahora.es, 2 de noviembre de 2006. Consultado en: 27-02-07.

http://www.canariasahora.com/portada/editar_noticia.asp?idnoticia $=84384 \&$ idtemagener $a l=13$

Dans, Enrique. "Algo más que noticias. Cómo se enfrenta la prensa tradicional al fenómeno de la Web 2.0". En: PC Actual, 19 de octubre de 2006. Consultado en: 27-02-07. prensa-pca.pdf

El confidencial. "El confidencial refuerza su equipo y renueva la imagen para seguir creciendo". En: El confidencial, 11 de septiembre de 2006. Consultado en: 27-02-07.

http://www.elconfidencial.com/noticias/noticia. asp $?$ id $=17014$ \& edicion $=11 / 09 / 2006 \&$ pass

El correo gallego. "El Grupo Correo Gallego renueva la edición digital y amplía contenidos". En: El correo gallego, 1 de mayo de 2006. Consultado en: 27-02-07.

http://www.elcorreogallego.es/index.php?idMen $u=130 \&$ idNoticia $=40020$

El mundo.es. "Conozca el nuevo diseño de elmundo.es". En: El mundo.es, 7 de junio de 2006. Consultado en: 27-02-07.

http://www.elmundo.es/elmundo/2006/graficos/ jun/s1/tuto_1024.html http://profesores.ie.edu/enrique_dans/download/ 
El pais.com. "La 'web' de EL PAÍS cambia de nombre, estrena diseño y da más protagonismo a los usuarios". En: El pais.com, 19 de noviembre de 2006. Consultado en: 27-02-07.

http://www.elpais.com/articulo/sociedad/ web/PAiS/cambia/nombre/estrena/dise no/da/protagonismo/usuarios/elpporsoc/ 20061119elpepisoc_9/Tes/

El periodico.com. "El periódico actualiza su página web". En: El periodico.com, 6 de septiembre de 2006. Consultado en: 27-02-07.

http://www.elperiodico.com/default. asp?idpublicacio_PK=46\&idioma $=C A S \&$ idnot cia_PK=337086\&idseccio_PK=1012

Guallar, Javier. "Prensa digital en 2006". En Baiget, Tomàs, Eds. Anuario ThinkEPI 2007, Barcelona: EPI SCP, 2006, pp. 106-117. Consultado en: 27-02-07.

http://eprints.rclis.org/archive/00008388/01/ AnuarioThinkEPI2007-Guallar-Prensa-digitalen-2006.pdf

La vanguardia.es. "La vanguardia.es mejora la consulta de la edición impresa y da acceso libre a la actualización las 24 horas del día”. En: $L a$ vanguardia.es, 13 de junio de 2006. Consultado en: 27-02-07. http://www.lavanguardia.es/lv24h/20060613/51 273454110.htm

Lafuente, Gumersindo. "El sitio de los lectores". En: El mundo.es, 8 junio de 2006. Consultado en: 27-02-07.

http://www.elmundo.es/elmundo/2006/06/06/comunicacion/1149625068.html

Margaix-Arnal, Dídac. "Conceptos de web 2.0 y biblioteca 2.0: origen, definiciones y retos para las bibliotecas actuales". En: El profesional de la información, 2007, marzo-abril, v. 16, n. 2, pp. 95-106.

Noguera-Vivo, José-Manuel. "Cibermedios nacionales y fuentes rss: una oportunidad por descubrir". En: Sabés, Fernando (ed.). Análisis y propuestas en torno al periodismo digital. Zaragoza: Asociación de la Prensa de Aragón, 2006, pp. 158-166. Consultado en: 27-02-07.

http://www.congresoperiodismo.com/alisis $\% 20$ y\%20propuestas $\% 20$ en $\% 20$ torno $\% 20$ al $\% 20$ peri odismo\%20digital.pdf

OneStat.com. "Screen resolution 800 x 600 significantly decreased for exploring the internet according to OneStat.com". En: OneStat.com, 25 de junio de 2006. Consultado en: 27-02-07. http://www.OneStat.com/html/abouts_pressbox43-screen-resolutions.html
Serrano-Cobos, Jorge. "Pasado, presente y futuro de la Web 2.0 en servicios de información digital". En: BiD: textos universitaris de biblioteconomia i documentació, desembre 2006, núm. 17. Consultado en: 27-02-07.

http://www2.ub.edu/bid/consulta_articulos. php? fichero $=17$ serra $2 . h t m$

Varela, Juan. Periodismo 3.0, la socialización de la información. Última actualización en: 1301-07. Consultado en: 27-02-07.

http://www.escolar.net/wiki/index.php/Periodismo_3.0\%2C_la_socializaci\%C3\%B3n_de_la_ informaci\% $\mathrm{C} 3 \% \mathrm{~B} 3 \mathrm{n}$

20 minutos.es. "Hemos cambiado, ¿cómo lo ves?". En: 20 minutos.es, 20 de junio de 2006 Consultado en: 27-02-07.

http://www.20minutos.es/noticia/132853/0/diseno/web/cambio/

\section{Javier Guallar}

El periódico de Catalunya, departamento de documentación.

Universitat de Barcelona, Facultat de biblioteconomia i documentació. jguallar@gmail.com

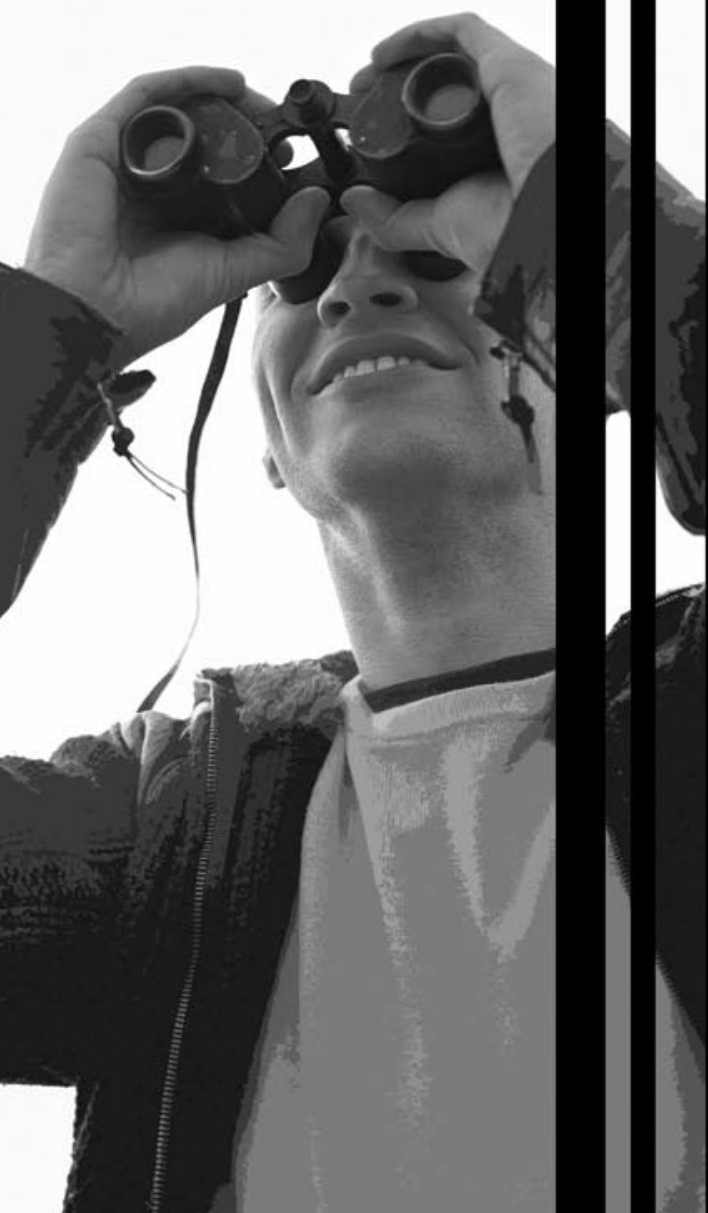

\section{MÁSTER EN DOCUMENTACIÓN DIGITAL}

\section{Máster y Programas de posgrado online}

Arquitectura de la información en sitios web. - Organización de la información y sistemas de gestión de bases de datos. - Buscadores y posicionamiento web. - Documentación audiovisual. - Gestión de proyectos en centros de información. - Archivística digital. - Gestión de contenidos y gestión del conocimiento. - Bibliotecas digitales. - Usabilidad.

Dirección: Cristòfol Rovira y Lluís Codina

Calendario: oct 07 - jun 08

www.documentaciondigital.org

\section{MÁSTER EN BUSCADORES}

\section{POSICIONAMIENTO, MARKETING ONLINE Y VIGILANCIA TECNOLÓGICA} Máster y Programas de posgrado online

El objetivo general del curso es proporcionar una sólida formación sobre todos los aspectos relacionados con los buscadores de Internet en sus diferentes facetas: herramientas de recuperación de información, medios privilegiados para el marketing online, instrumentos para implementar programas de vigilancia tecnológica e inteligencia competitiva.

Dirección: Cristòfol Rovira y Lluís Codina

Calendario: nov 07 - jun 08

www.masterenbuscadores.com

\section{Colaboran:}

Observatorio de Comunicación Científica - UPF Instituto Universitario de Lingüística Aplicada - UPF DOC 6 Consultores de Recursos de Información

Infórmate www.idec.upf.edu | info@idec.upf.edu | Tel. 935421850 | Balmes 132, Barcelona 